\title{
Unusual Etiology of Lumbar Radiculoplexopathy
}

\section{Kenneth W. Rabe, DO, Geoffrey K. Seidel, MD INTRODUCTION}

A patient presented to the authors' clinic with a unilateral single-level lumbar radiculopathy. No compressive etiology of the radiculopathy could be established with anatomical imaging of the spine. The patient experienced progressive neurophysiologic losses on serial examination despite treatment. A more aggressive workup was undertaken and yielded a final diagnosis of a lumbar radiculoplexopathy caused by a malignant peripheral nerve sheath tumor. The initial presentation is described, and the differential diagnosis, the diagnostic evaluation, and the management of this rare cause of a lumbar radiculoplexopathy are reviewed.

\section{CASE REPORT}

A 35-year-old woman presented with progressive right buttock pain, foot drop, and L5 sensory loss. Her medical history was significant for a uterine sarcoma, occurring in 1991, which was treated with a total abdominal hysterectomy and left salpingo-oophorectomy along with left pelvic radiation. In 1997 and 1998, on 2 separate occasions, metastatic disease to the lung was found. In both instances, these metastatic recurrences of her uterine sarcoma were successfully treated with chemotherapy and a partial lung wedge resection and left lung lower lobectomy in 1997 and 1998, respectively. She reported mild distal sensory impairment after previous uterine sarcoma-related chemotherapy. Sensory peripheral polyneuropathy was not documented in an electrodiagnostic study performed 6 months before the authors' initial consultation.

Before the patient's initial presentation to the authors, a previous workup included lumbar radiography, lumber magnetic resonance imaging (MRI) without contrast, and electrodiagnostic (Edx) testing. Findings at lumbar radiography were normal. The MRI did not reveal mass, disk herniation, or other compressive processes. Previous Edx showed normal nerve conduction studies of the right peroneal motor and right sural sensory nerves with acute denervation ( 2 to $3+$ positive waves and fibrillation potentials) of the right anterior tibialis, extensor hallicus longus, peroneus longus, tibialis posterior, and gluteus medius but no motor unit abnormalities were described on needle electromyography (EMG). Normal results were described for needle examination in right vastus medialis, adductor longus, and medial gastrocnemius muscles. The electromyographer reported a conclusion of an acute severe right L5 radiculopathy with a "paucity of paraspinal changes, cannot rule out lumbosacral plexopathy though this is much less likely."

The initial physical examination revealed 3/5 right anterior tibialis and right gluteus medius, and 4/5 extensor hallicus longus muscle weakness. All remaining right lower limb muscles were 5/5. The patient had normal strength of bilateral upper as well as the left lower limb musculature. The right gluteal area, posterior thigh, and anterior calf revealed visible atrophy. Her sensory examination revealed a report of $90 \%$ reduction of sensation isolated in the right L5 dermatome when subjective questioning was used as compared with the opposite limb. She reported bilateral impairment of distal vibratory sensation with normal proprioception. She exhibited a decreased sensory perception to pinwheel in a distal symmetrical sock-like distribution with transition at the malleoli. Her gait revealed a right Trendelenburg, pelvic drop, and increased lateral deviation. Muscle stretch reflexes were symmetric with Achilles graded 1/4 and quadriceps graded 2/4. Right medial hamstring reflex was absent and graded $2 / 4$ at the left.
K.W.R. Rehabilitation Institute of Michigan/ Wayne State University, 261 Mack Ave., Detroit, Ml 48201. Address correspondence to K.W.R. k_rabe_do@yahoo.com. Disclosures: nothing to disclose.

G.K.S. Wayne State University, Department of Physical Medicine and Rehabilitation, Detroit, MI.

Disclosures: nothing to disclose.

Submitted for publication May 20, 2008; accepted January 1, 2009. 
Table 1. Right lower limb needle EMG results

\begin{tabular}{lllclcc}
\hline Muscle & PW & Fib & Rec & Dur & Amp & Poly \\
\hline AT & $4+$ & $4+$ & 0 & 0 & 0 & 0 \\
EHL & $3+$ & $3+$ & -4 & $\mathrm{NL}$ & $\mathrm{NL}$ & $\mathrm{NL}$ \\
BSH & $3+$ & $3+$ & -4 & $1+$ & $1+$ & $1+$ \\
GMed & 0 & 0 & -4 & $2+$ & $2+$ & $2+$ \\
SM & 0 & 0 & -2 & $1+$ & $1+$ & $1+$ \\
VM & 0 & 0 & $\mathrm{NL}$ & $\mathrm{NL}$ & $\mathrm{NL}$ & $\mathrm{NL}$ \\
GS & 0 & 0 & $\mathrm{NL}$ & $\mathrm{NL}$ & $\mathrm{NL}$ & $\mathrm{NL}$ \\
GMax & 0 & 0 & $\mathrm{NL}$ & $\mathrm{NL}$ & $\mathrm{NL}$ & $\mathrm{NL}$ \\
AM & 0 & 0 & $\mathrm{NL}$ & $\mathrm{NL}$ & $\mathrm{NL}$ & $\mathrm{NL}$ \\
L2-4 & 0 & 0 & & & & \\
L5-S1 & $2+$ & $2+$ & & & & \\
\hline
\end{tabular}

$\mathrm{AT}=$ anterior tibialis, $\mathrm{EHL}=$ extensor hallicus longus, $\mathrm{BSH}=$ biceps femoris short head, GMed $=$ gluteus medius, $\mathrm{SM}=$ semimembranosus, $\mathrm{VM}=$ vastus medialis, GS = medial and lateral gastrocnemius, GMax = gluteus maximus, $\mathrm{AM}=$ adductor magnus, $\mathrm{L} 2-\mathrm{S} 1$ = lumbosacral paraspinals, NL $=$ normal.

With a presumed diagnosis of acute radiculopathy, oral steroid treatment was instituted. The steroid regimen improved her pain, but weakness continued to progress in the right lower limb, causing further gait impairment. Repeated lumbar MRI with gadolinium did not reveal recurrence/ metastases of uterine sarcoma. A total body bone scan did not reveal bony metastases. On the basis of the diagnostic testing to this point, there was no clear anatomical etiology to explain the neurologic deficits and previous Edx results.

A new Edx study was performed, and it was suggestive of an isolated L5 radiculopathy with paraspinal denervation (Tables 1 and 2). The right lower limb was cool at $27^{\circ} \mathrm{C}$ despite warming attempts. Right sural sensory study showed prolonged distal latency with a borderline amplitude. Right peroneal and tibial motor conduction studies were within normal limits. (In retrospect, left lower limb sensory and motor nerve conduction studies would have been beneficial for side-to-side comparison to determine a postganglionic lesion; however, at that time there was a presumed diagnosis of an isolated radiculopathy and the patient had no left lower-limb complaints.) The authors considered an MRI of the pelvis/lumbar plexus with contrast but, after oncology consultation, positron emission tomography/computed tomography (PET/CT) of the chest, abdomen, and pelvis was performed and revealed an abnormal focus of activity in the right hemipelvis. The reviewing radiologist was concerned about a colonic source and recommended colonoscopy. Colonoscopy did not reveal any abnormality.
The differential diagnosis of a noncompressive, inflammatory radiculopathy was considered: viral etiology, diabetic or nondiabetic plexopathy, autoimmune disorders, "chemical" radiculitis secondary to a degenerative disk/anular tear, etc. A metabolic workup revealed the following normal and negative values: The patient had normal renal, hepatic, endocrine (thyroid and blood sugars [glucose tolerance test]), erythrocyte sedimentation rate, antinuclear antibodies, rheumatoid factor, serum protein electrophoresis, creatine phosphokinase, alkaline phosphotase, VDRL test, vitamin B12, and folate.

In addition to the oral steroids instituted for a presumed inflammatory radiculopathy, the patient was prescribed the antiepileptic agent gabapentin. Medications relieved her pain symptoms, but the patient continued to have neurophysiologic losses evident on serial motor examinations. During the workup period, the right anterior tibialis and extensor hallicus longus declined to $1 / 5$ and right gluteus medius declined to $2 / 5$. In light of the progressive loss of motor function limited to the right lower limb with continued completely normal examinations of both upper limbs and the left lower limb, it was concluded that the progressive decline in function was not a generalized systemic process.

Because of the rapid progressive deterioration and the positive PET scan results, a recurrence of cancer or some other pathological process involving the plexus was suspected. After discussion with the oncologist, the patient was referred to neurosurgery for evaluation and consideration of lumbar plexus/sciatic nerve exploration.

The sciatic nerve was explored at the sciatic notch and was found to be markedly thickened at this location. Intraoperative electrical stimulation was performed, revealing that the medial portion of the sciatic nerve was functional, but no response was obtained with stimulation of the lateral portion of the sciatic nerve. A biopsy was obtained from the lateral aspect of the sciatic nerve, but proximal exploration to determine the extent of the lesion was not performed at that time. Sections revealed cellular proliferation of poorly differentiated spindle cells infiltrating the nerve, forming sheets and ill-defined fascicles and bounded by a perineurial connective tissue layer. Nuclei showed a moderate degree of pleomorphism with coarsely clumped chromatin. Immunohistochemical stain for vimentin was diffusely positive, p53 showed nuclear staining in many cells, CD10 was positive in patchy areas, and CD57 was positive in scattered tumor cells. Immunohistochemical stains for S100, C-kit, CD45, and

Table 2. Nerve conduction results

\begin{tabular}{llcccc}
\hline Side & Nerve & Segment $(\mathbf{c m})$ & Latency (ms) & Amplitude & Velocity (m/s) \\
\hline$R$ & Sural (s) & 14 & 6.4 & $9 \mu \mathrm{V}$ \\
$R$ & Peroneal (m), ankle & 9 & 5.7 & $0.8 \mathrm{mV}$ & $0.7 \mathrm{mV}$ \\
& below fibular head & 24 & 11.7 & $0.6 \mathrm{mV}$ & 40 \\
& above fibular head & 10 & 13.2 & $13.4 \mathrm{mV}$ & 66 \\
$R$ & Tibial (m), ankle & 8 & 5.0 & $10.1 \mathrm{mV}$ \\
& Popliteal & 34 & 12.6 & 44 \\
\hline
\end{tabular}

Temperature of $27^{\circ} \mathrm{C}$. Sensory latency measured to peak. Peroneal motor conduction to extensor digitorum brevis; tibial motor conduction to adductor hallicus 
EMA were negative. These results were consistent with a high-grade neurosarcoma with an unusually high proliferative index best classified as a malignant peripheral nerve sheath tumor involving the peroneal division of the sciatic nerve.

After the diagnosis of a malignant peripheral nerve sheath tumor was established, it was recognized that complete removal of the tumor was required. The patient was referred to a regional tertiary care facility, where she underwent radiation therapy and 2 courses of chemotherapy to minimize the size of the tumor. She then underwent neurosurgical resection of the involved portions of the sciatic nerve. The surgical exploration continued proximally to involve the lumbosacral plexus and then further exploration extended proximal to the dorsal and ventral rami of the L5 spinal nerve root. Biopsy results confirmed the bulk of involvement of the neurosarcoma in the lumbar plexus. Clean margins were established, no further chemotherapy or radiation therapy was performed, and the patient was discharged home.

\section{DISCUSSION}

Given the history of previous uterine sarcoma, a recurrence of metastatic uterine sarcoma was the primary concern until proven otherwise. These tumors have a tendency to recur after removal and metastasize through the bloodstream, primarily to the lungs and brain, and less frequently to bone. The 5-year survival rate averages $40 \%$ for patients with uterine sarcomas that disseminate throughout the abdominal cavity [1]. Neoplastic lumbosacral radiculoplexopathies have been reported by direct perineurial spread into the lumbosacral plexus [2], but in this case, no recurrence of uterine sarcoma was found.

Once a recurrence of the uterine sarcoma was excluded from the differential diagnosis, treatment of inflammatory radiculopathy was initiated. This diagnosis was supported by the absence of a compressive lesion from a lumbar disc herniation, no neuroforaminal narrowing, no nerve root sheath cyst, no facet joint cyst, no metastatic mass lesion, and the fact that the patient's pain symptoms improved temporarily with oral steroids. A literature review failed to provide a frequency of single root-level noncompressive inflammatory lumbar radiculopathy. In the authors' experience, patients with inflammatory, noncompressive radiculopathies most often have multilevel nerve root involvement and do not present as an isolated radiculopathy.

Because of an absence of a compressive lesion on MRI and because the administration of oral steroids did not alter or slow the progressive neurophysiologic loss (which would be the case in an inflammatory radiculopathy), the focus was shifted to the lumbosacral plexus. MRI of the pelvis with gadolinium before the neurosurgical evaluation would have been reasonable based on electrodiagnostic findings and 2 normal MRIs of the lumbosacral spine. However, in this case, time was of the essence because the patient's clinical functional status was rapidly deteriorating. None of the clinicians involved in her care-a physiatrist, oncologist, and neuro- surgeon-believed a pelvic MRI was necessary when making a management decision in this particular case, but in general pelvic MRI is a reasonable part of the workup. Lumbosacral plexopathies are difficult to accurately diagnose [3]. Technology has yet to advance to consistently yield reliable results with MRI imaging of the pelvis. MR neurography could have been a viable option because it would provide better detail of the plexus; however, this test is unavailable in our practicing area. Most often an MRI of the lumbar spine has the highest yield in establishing a correlating compressive cause of radiculopathy. The contrary scenario occurred in this case, in which progressive EMG abnormalities were paramount.

Although fibrillation potentials may be present as falsepositive results, the surgical exploration revealed that paraspinals were likely denervated from tumor involvement of dorsal rami. The authors thought that the significant denervation potentials found in this case on EMG were true neurophysiological findings and not false-positive findings because no abnormal denervation potentials were found at other levels of the spine. Before the authors were aware of the plexus lesion and biopsy results, it was considered that an inflammatory process could explain the denervation of the paraspinal musculature. Unfortunately, treatment for an inflammatory process was unsuccessful in this case. A concept of retrograde axonal loss from a plexus lesion was considered and discounted as an explanation for the paraspinal denervation because it was thought that distal axonal loss would not impact proximal paraspinal alpha motor neurons. The authors later determined that the proximal spread of the neurosarcoma to the dorsal rami resulted in the paraspinal denervation.

Paraspinal muscles are innervated by the dorsal primary rami of the spinal nerve that immediately exit the neural foramen. If there are abnormalities on EMG examination, the lesion must be at least as far proximal as the root level [4]. The patient's neurosarcoma originated in the lumbosacral plexus and migrated proximal to the dorsal and ventral spinal root junction to involve the alpha motor neurons of the ventral spinal root. The axonal loss evident in the paraspinal muscles at the $\mathrm{L} 5$ level was caused by proximal migration of the nerve sheath tumor.

The authors did not perform a left sural sensory evaluation during the Edx evaluation but, in retrospect, this study would have been helpful to gain further insight to a plexus predominant lesion. A low-amplitude unilateral sural sensory result would have suggested a postganglionic lesion and likely further clarified the clinical presentation.

This case presents a rare nerve sheath tumor. In a 30-year study of 397 peripheral nerve sheath tumors by Kim et al [5], 361 cases were benign and 36 were malignant, of which 28 were neurosarcomas and 8 were other sarcomas (fibro-, spindle cell, synovial, and perineurial sarcomas), and malignant peripheral nerve sheath tumors are associated with a high degree of morbidity and mortality. The 5- and 10-year survival rates for patients with malignant peripheral nerve sheath tumors were found to be $52 \%$ and $34 \%$, respectively, with a goal of treatment being complete resection with neg- 
ative margins and possible adjuvant irradiation, intraoperative electron irradiation, and brachytherapy [6]. The role of chemotherapy has yet to be determined but may be of benefit in salvaging treatment failures $[7,8]$.

\section{CONCLUSION}

In this case, there was no clear explanation for the clinical presentation of foot drop from an apparent L5 radiculopathy; the authors suspected metastatic uterine sarcoma and persisted in the workup because of the patient's deteriorating condition on serial examinations. This case has educational value for physicians involved in the diagnosis and treatment of radiculopathies, plexopathies, and other peripheral nerve lesions. This patient presented with profound progressive motor and sensory deficits whose etiology could not be established through standard workup. In cases such as these, a physiatrist must expand the physical examination and broaden the differential diagnosis. Although this presentation is a rare occurrence, the authors hope this case will help organize and prioritize the workup of patients who present with neurological symptoms and continue to decline despite standard treatment and workup. A physician should not stop with an assumed diagnosis of an inflammatory radiculopathy or accept an idiopathic label in the presence of a deteriorating clinical course; in these rare cases, a malignant peripheral nerve sheath tumor could be considered.

\section{REFERENCES}

1. Crum CP. The Female Genital Tract. In: Cotran RS, Kumar V, Collins T, eds. Robbins Pathologic Basis of Disease, 6th ed. Philadelphia: W. B. Sauders Company; 1999, 1064-1065.

2. Ladha SS, Spinner RJ, Suarez GA, Amrami KK, Dyck PJ. Neoplastic lumbosacral radiculoplexopathy in prostate cancer by direct perineural spread: an unusual entity. Muscle Nerve. 2006;34:659-665.

3. Tavee J, Mays M, Wilbourn AJ. Pitfalls in the electrodiagnostic studies of sacral plexopathies. Muscle Nerve 2007;35:725-729.

4. Dumitru D, Amato AA, Zwarts MJ, eds. Electrodiagnostic Medicine, 2nd ed. Philadelphia: Hanley \& Belfus, Inc., 2002, 570-571, 713-776.

5. Kim DH, Murovic JA, Tiel RL, Moes G, Kline DG. A series of 397 peripheral neural sheath tumors: 30 year experience at Louisiana State University Health Sciences Center. J Neurosurg 2005;1-2:246-255.

6. Wong WW, Hirose T, Scheithauer BW, Schild SE, Gunderson LL. Malignant peripheral nerve sheath tumor: analysis of treatment outcome. Int J Radiat Oncol Biol Phys 1998;42:351-360.

7. Wanebo JE, Malik JM, VandenBerg SR, Wanebo HJ, Driesen N, Persing JA. Malignant peripheral nerve sheath tumors. A clinicopathologic study of 28 cases. Cancer 1993;71:1247-1253.

8. Rawal A, Yin $\mathrm{Q}$, Roebuck $M$, et al. Atypical and malignant peripheral nerve sheath tumors of the brachial plexus: Report of three cases and review of the literature. Microsurgery 2006;26:80-86. 